Original Article

\title{
The effect of external cues with vibratory stimulation on spatiotemporal gait parameters in chronic stroke patients
}

\author{
Jae Myoung Park, PT, MS ${ }^{1)}$, Hee Sung Lim, PT, MS ${ }^{1)}$, Chang Ho Song, PT, PhD ${ }^{1)^{*}}$ \\ 1) Department of Physical Therapy, Sahmyook University: 26-21 Gongneung2-dong, Nowon-gu, Seoul \\ 139-742, Republic of Korea
}

\begin{abstract}
Purpose] The aim of current study was to investigate the effect of external cues using vibratory stimulation on spatiotemporal gait parameters in patients with chronic stroke. [Subjects] Thirty patients, who had suffered a stroke, were selected from general hospitals in Seoul, Republic of Korea. [Methods] Each participant was subjected to six walking trials: three trials with vibratory stimulation of the tibialis anterior muscle and three trials without any stimulation. The spatiotemporal gait parameters were measured with a GAITRite system. [Results] The global gait parameters - velocity and cadence - were significantly more increased, and the temporal parametersstep time, stride time, and double limb support - were significantly more decreased with vibratory stimulation of the tibialis anterior muscle than with no stimulation. While single limb support was increased under vibratory stimulation, the difference was not significant between the two groups. The spatial gait parameter - stride lengthshowed a significant improvement; however, there was no significant enhancement in gait symmetry with regard to step length and step time [Conclusion] These results indicate that the application of external cues using vibratory stimulation during gait may control gait parameters and improve gait performance. Thus, this intervention could be used for gait rehabilitation in chronic stroke patients.

Key words: Vibratory stimulation, Gait, Stroke
\end{abstract}

(This article was submitted Jul. 9, 2014, and was accepted Aug. 24, 2014)

\section{INTRODUCTION}

After a stroke, gait is an important determinant of functional ability in the activities of daily living and for an independent life ${ }^{1)}$. In general, stroke patients continue to have walking difficulties, including reductions in their gait velocity and endurance caused by the weakness of muscles and deterioration of voluntary control function ${ }^{2}$. Weakness of muscles involved in dorsiflexion or lack of voluntary control causes foot drop because of the lack of sufficient dorsiflexion in the swing phase ${ }^{3)}$.

Therapeutic approaches used in clinical settings to improve foot drop in stroke patients include the application of ankle-foot orthoses (AFO) and functional electrical stimulation ${ }^{4}$. These arbitration methods are used to correct foot drop and improve the functional outcome of rehabilitation in stroke patients. However, because stroke patients do not actively use hemiplegic feet, muscular control of contraction is challenging ${ }^{4,5)}$.

Various types of external cues provide information to

*Corresponding author. Chang Ho Song (E-mail: chsong@ syu.ac.kr)

(C2015 The Society of Physical Therapy Science. Published by IPEC Inc. This is an open-access article distributed under the terms of the Creative Commons Attribution Non-Commercial No Derivatives (by-ncnd) License $<$ http://creativecommons.org/licenses/by-nc-nd/3.0/> . control gait parameters such as step frequency or step amplitude $^{6}$. Several studies have assessed the use of auditory and visual external cues to improve gait and overcome functional limitations due to specific damage in stroke patients. Acoustic signals control temporal gait parameters such as cadence by providing external rhythms to compensate for deficient internal signals from the basal ganglia, whereas visual signals control spatial gait parameters such as step and stride lengths ${ }^{7}$. In patients with chronic stroke, the application of external signals such as rhythmic auditory stimulation has resulted in improved spatiotemporal gait parameters through anticipatory postural control and sequential activation of muscle contraction ${ }^{8)}$. Therefore, controlled application of acoustic and visual signals is effective in improving gait parameters. However, these signals cannot be applied without the help of an assistant; moreover, it is difficult to use these two signals for gait training in the presence of small noises or dark lighting ${ }^{9)}$.

To overcome these problems, Van et al. ${ }^{9)}$ suggested the application of somesthetic signals using vibration machines, which have a significant effect on gait parameters, as a practical alternative stimulus to acoustic and visual signals for improvement of gait in patients with Parkinson's disease. In several studies, vibratory stimulation was used to improve postural stability, improve muscular strength by providing information for proprioceptive sensibility, and reduce spasticity by somesthetic stimulation ${ }^{10,11)}$. In patients with Parkinson's disease, application of external signals such as 
vibratory stimulation has resulted in improved spatiotemporal gait ${ }^{12)}$.

However, in most of the studies involving stroke patients, vibratory stimulation was physiologically applied through activation of muscle spindles and primary afferent fibers, and external cues were applied using visual and auditory stimuli. In the current study, we examined the effect of applying external cues using vibratory stimulation during the gait cycle on spatiotemporal gait parameters in chronic stroke patients with deteriorated gait ability due to foot drop.

\section{SUBJECTS AND METHODS}

For the present study, we recruited a total of thirty outpatients and inpatients (18 men and 12 women) with chronic stroke. Nineteen patients had left hemiplegia, and eleven had right hemiplegia. The average age of the participants was 58; average height of the participants was $164.4 \mathrm{~cm}$, and average weight of the participants was $65.5 \mathrm{~kg}$. The average duration of disease was 17 months, and the average Korean minimental state examination (K-MMSE) score of the participants was 28 points (Table 1). The inclusion criteria of the study were as follows: 6 months since the diagnosis of stroke, K-MMSE $>24$ points, ability to walk for a minimum of 10 $\mathrm{m}$, no problem in gait by contracture of the foot and ankle, and no musculoskeletal disease. Patients with gait problems due to orthopedic surgery or disability of the limbs and those with vestibular organ or cerebellum-related diseases were excluded. The study was conducted after obtaining approval from the ethics committee of Sahmyook University, and all participants provided written informed consent.

For the analyses of spatiotemporal gait parameters in stroke patients, we used foot switches and vibration machines to provide a vibratory stimulus. A gait analysis system (GAITRite, CIR Systems, Inc., Sparta, NJ, USA) was used to measure the spatial and temporal parameters of gait ${ }^{13)}$.

Based on the verbal signals of an inspector, participants completed 6 walking trials at their most comfortable gait velocity on the GAITRite walkway under two conditions: with vibratory stimulation and without vibratory stimulation. First, the vibration machines were attached to the center of the tibialis anterior muscle on the affected side of the patients, and then foot switches were attached to the heels on their unaffected sides. Vibratory simulation was applied to the tibialis anterior muscle of the affected side starting from the initial contact phase at the beginning of stance when the heel just touched the ground. A 5-minute break was given to the participants after three walking trials. Gait with vibratory stimulation applied to the tibialis anterior muscle and general gait (without stimulation) were randomly measured three times, and the mean values were used for analyses.

The GAITRite walkway was used to measure spatial and temporal gait parameters. The inter-rater reliability of the GAITRite is $r=0.90$, and the inter-rater correlation coefficient $(\mathrm{ICC}=0.99$ ) is more than $r=0.96$. The gait analysis system has 16,128 sensors, which are vertically arranged in the walking plate at intervals of $1.27 \mathrm{~cm}$. The active area is $61 \mathrm{~cm}$ wide and $427 \mathrm{~cm}$ long. Using this system, the following measures were analyzed: the gait velocity and cadence;
Table 1. Common characteristics of the participants

\begin{tabular}{|c|c|c|}
\hline \multirow{2}{*}{ Characteristics } & \multicolumn{2}{|l|}{ Values $(\mathrm{N}=30)$} \\
\hline & Mean & $\mathrm{SD}$ \\
\hline Gender (male/female) & $18(60.0 \%) / 12(40.0 \%)$ & \\
\hline Age (yrs) & 58.0 & 10.0 \\
\hline Height (cm) & 164.4 & 7.7 \\
\hline Weight (kg) & 65.5 & 8.1 \\
\hline Paretic side (left/right) & $19(63.3 \%) / 11(26.7 \%)$ & \\
\hline Poststroke duration (month) & 17.0 & 9.6 \\
\hline Mini-mental state examination & 28.0 & 2.5 \\
\hline
\end{tabular}

temporal gait parameters, including stride time, step time, single support percentage of cycle, and double support percentage of cycle; and spatial gait parameters, including step and stride lengths.

The gait symmetry ratio was used to examine the symmetry of spatiotemporal gait of stroke patients according to the study of Patterson ${ }^{14)}$.

Symmetry ratio $=\left|\frac{\text { paretic side }(\text { parameter value })}{\text { nonparetic side }(\text { parameter value })}\right|$

The absolute values were used to calculate the gait symmetry ratio. Values of this ratio closer to 1 indicate greater increases in gait symmetry, and values farther from 1 indicate decreased gait symmetry. If the symmetry ratio is higher, the values of the affected side are higher than those of the unaffected side. Conversely, if the symmetry ratio is lower, the values of the affected side are lower than those of the unaffected side.

All statistical analyses were performed using SPSS Ver. 18.0. Normality of the study population was examined by the Shapiro-Wilk test. The independent t-test was performed to examine the homogeneity of the participants. The paired t-test was carried out to examine changes in spatiotemporal gait parameters between general gait and vibration-stimulated gait. A significance level of $\mathrm{p}=0.05$ was used.

\section{RESULTS}

The spatiotemporal parameters of gait after the application of vibratory stimulation were compared with those of general gait (Table 2). The gait velocity under vibratory stimulation of the tibialis anterior muscle $(50.22$ $\pm 17.42 \mathrm{~cm} / \mathrm{s}$ ) was significantly higher than that of general gait $(46.59 \pm 15.09 \mathrm{~cm} / \mathrm{s} ; \mathrm{p}<0.05)$. Similarly, the gait cadence under vibratory stimulation of the tibialis anterior $(79.26 \pm 15.76 \mathrm{step} / \mathrm{min})$ was significantly higher than that of general gait $(76.32 \pm 13.13$ step/min; $\mathrm{p}<0.05)$. Under vibratory stimulation, the step time of the unaffected and affected sides significantly decreased (from $0.75 \mathrm{~s}$ to $0.03 \mathrm{~s}$ and from $0.89 \mathrm{~s}$ to $0.87 \mathrm{~s}$, respectively; $\mathrm{p}<0.05$ ). The stride time of gait significantly decreased from $1.37 \mathrm{~s}$ to $0.77 \mathrm{~s}$ (p $<0.05$ ). Compared with general gait, the single lower limb support rate of the unaffected and affected sides under vibratory stimulation increased from 32.23 to $36.52 \%$ and from 
Table 2. Spatiotemporal parameters of gait under the two conditions $(\mathrm{N}=30)$

\begin{tabular}{|c|c|c|c|c|}
\hline \multirow{2}{*}{ Parameters } & \multicolumn{2}{|c|}{ VS gait } & \multicolumn{2}{|c|}{ Non-VS gait } \\
\hline & Mean & SD & Mean & SD \\
\hline Gait velocity $(\mathrm{cm} / \mathrm{s})$ & $50.22 *$ & 17.42 & 46.59 & 15.09 \\
\hline Cadence (step/min) & $79.26^{*}$ & 15.78 & 76.32 & 13.12 \\
\hline \multicolumn{5}{|l|}{ Step time } \\
\hline Affected side & $0.87 *$ & 0.24 & 0.89 & 0.22 \\
\hline Less affected side & $0.72 *$ & 0.19 & 0.75 & 0.17 \\
\hline Stride time & $0.65^{*}$ & 0.28 & 1.37 & 0.51 \\
\hline \multicolumn{5}{|l|}{ Single lower limb support rate $(\% \mathrm{GC})$} \\
\hline Affected side & 27.93 & 7.58 & 27.32 & 7.58 \\
\hline Less affected side & $36.52 *$ & 5.44 & 35.32 & 5.13 \\
\hline Double limb support rate (\%GC) & $36.80^{*}$ & 7.04 & 38.50 & 7.23 \\
\hline \multicolumn{5}{|l|}{ Step length $(\mathrm{cm})$} \\
\hline Affected side & $38.42 *$ & 8.66 & 36.78 & 7.76 \\
\hline Less affected side & 35.63 & 8.05 & 34.76 & 7.42 \\
\hline Stride length $(\mathrm{cm})$ & $69.11^{*}$ & 24.18 & 66.78 & 22.65 \\
\hline \multicolumn{5}{|l|}{ Gait symmetry } \\
\hline \multicolumn{5}{|l|}{ Temporal gait symmetry } \\
\hline Symmetry ratio & 1.21 & 0.18 & 1.20 & 0.19 \\
\hline Gait asymmetry & 18.90 & 13.78 & 17.70 & 14.21 \\
\hline \multicolumn{5}{|l|}{ Spatial gait symmetry } \\
\hline Symmetry ratio & 1.10 & 0.02 & 1.08 & 0.19 \\
\hline Gait asymmetry & 15.14 & 12.12 & 13.43 & 15.81 \\
\hline
\end{tabular}

VS gait: gait with vibratory stimulation applied to the tibialis anterior muscle of the affected side; NonVS gait: gait without vibratory stimulation

*Significantly different compared with the Non-VS gait condition $(\mathrm{p}<0.05)$

27.32 to $27.93 \%$, respectively, but no significant difference was observed in the latter case. Compared with general gait, the double limb support rate under vibratory stimulation was significantly decreased from 38.5 to $36.8 \%(\mathrm{p}<0.05)$.

The step lengths of the affected and unaffected sides under vibratory stimulation of the tibialis anterior muscle were $38.42 \mathrm{~cm}$ and $35.63 \mathrm{~cm}$, respectively; that is, the step lengths were $0.87 \mathrm{~cm}$ and $1.63 \mathrm{~cm}$ higher than those of the affected and unaffected sides in general gait. However, these differences were not statistically significant. The stride length under vibratory stimulation $(69.11 \mathrm{~cm})$ was significantly higher than that in general gait $(66.78 \mathrm{~cm} ; \mathrm{p}<0.05)$.

Furthermore, the changes in gait symmetry were as follows: First, with regard to temporal gait symmetry, there were no significant differences between the gait symmetry ratios under vibratory stimulation $(1.21 \pm 0.18)$ and general gait (1.20 \pm 0.19$)$. Moreover, no significant differences were found in the gait asymmetry ratios under vibratory stimulation (18.90 \pm 23.78$)$ and general gait $(17.70 \pm 13.78)$. Next, with regard to spatial gait symmetry, no significant difference was found in the symmetry of step lengths between gait under vibratory stimulation $(1.10 \pm 0.20)$ and general gait $(1.08 \pm 0.19)$. Moreover, there was no significant difference in the asymmetry of step lengths between gait under vibratory stimulation $(15.14 \pm 12.12)$ and general gait $(13.43 \pm$ 15.81).

\section{DISCUSSION}

In this study, to increase the gait velocity and endurance of stroke patients who had reduced gait abilities, we used a gait analysis system to examine the effect of external cues applied using vibratory stimulation on the spatiotemporal parameters of gait.

The results of this study showed that gait velocity and cadence were more significantly improved under vibratory stimulation of the tibialis anterior muscle than under general gait conditions $(\mathrm{p}<0.05)$. This result is in accordance with those of previous studies, which reported that gait performance was improved under vibratory stimulation of the tibialis anterior muscle in stroke patients with deteriorated gait ability due to foot drop ${ }^{5,15)}$.

Most stroke patients show asymmetrical gait patterns due to reduction in cadence and low gait velocity ${ }^{16)}$, and weakness of the hemiplegic plantar flexors in the push-off stage of gait is considered the most important factor ${ }^{17}$ ). But the importance of strengthening the ankle dorsiflexors has recently been highlighted for improvement of gait velocity ${ }^{18}$. Laufer et al. argued that muscular strength of the plantar flexors of the ankle on the affected side has an effect on gait velocity ${ }^{19)}$. Therefore, induction of voluntary contraction of the ankle plantar flexors using external cues involving vibratory stimulation of the tibialis anterior muscle during gait cycle is thought to decrease the duration of the swing phase and 
increase gait velocity.

In this study, the step time of the unaffected and affected sides decreased significantly under vibratory stimulation of the tibialis anterior muscle $(\mathrm{p}<0.05)$. The stride time was also significantly decreased $(\mathrm{p}<0.05)$. The single lower limb support rate of the unaffected sides showed significant improvement $(\mathrm{p}<0.05)$, while no significant difference was found in the single lower limb support rate of the affected sides. The double limb support rate was significantly decreased $(\mathrm{p}<0.05)$.

Patterson et al. reported that an increase in cadence implied a decrease in step time of the two sides, and a decreased lower limb support rate can be regarded as a surrogate marker of improved balance control ${ }^{14)}$. Maki et al. argued that an increase in double support percentage of cycle is related to the fear of falling while walking ${ }^{20)}$. Based on these studies, it is believed that the increased cadence and gait velocity indicated a decreased step time for the two sides and that the decrease in double limb support rate implied an improvement in continuity and balance of gait in the stroke patients. The step lengths of the unaffected sides under vibratory stimulation of the tibialis anterior muscle increased, but no significant difference was noted; however, the step lengths of the affected sides were found to increase significantly. In addition, the stride lengths under vibratory stimulation of the tibialis anterior muscle were significantly increased ( $p$ $<0.05$ ). These results concur with those of previous crosssectional studies that examined the gait characteristics of stroke patients $^{21,22)}$.

Patterson et al. reported that the increase in stride lengths is due to the increase in step lengths of the two sides, and Balasubramanian et al. reported that the changes in the affected legs resulted in improved gait ability due to increased step lengths of the unaffected sides ${ }^{14,23)}$. Therefore, it is considered that application of vibratory stimulation during gait cycles increases the range of motion by inducing voluntary contraction of the ankle plantar flexors and that increases in step length of the affected side will cause a greater improvement in gait impulse to the front. In this study, no significant difference in spatial and temporal gait symmetry was found. It is possible that vibratory stimulation did not have an immediate significant effect on gait symmetry but may produce a significant effect over a longer period. Previous studies that showed a significant effect of external signals were conducted for a longer duration. In this study, the external cues with vibratory stimulation significantly improved gait parameters, including gait velocity and cadence, but did not have any effect on gait symmetry.

Kottink et al. argued that application of repetitive stimulation to muscles during the gait cycle for an extended period may promote functional recovery due to reinforcement of feedback to the brain. Therefore, vibratory stimulation is more effective in improving motor skill recovery ${ }^{24)}$. Previous studies have reported that passive and active movements strengthen the activity of the primary motor ${ }^{25,26)}$. Using functional magnetic resonance imaging (fMRI), Lotze et al. showed that the activity of the primary motor cortex is more significantly increased after active training compared with passive activity ${ }^{27)}$. In stroke patients, deterioration of gait ability is primarily caused by a lack of voluntary control of muscle contraction, thereby resulting in the loss of ability to control the timing and intensity of muscular activity ${ }^{1)}$. Selective muscular control, however, can be induced by external cues, such as vibratory stimulation, which cause active muscular contraction. An external cue provided at the proper time could improve the preparation procedures, which could lead to a more normal movement. Each different type of cue results in activation of a different strategy of motor control. The sensory cues allow the dorsolateral premotor control system (voluntary) to detouring the supplementary motor area's deficit (automatic movement), and external cues enable the premotor cortex to remain intact, rather than the basal ganglia/supplementary motor area circuit ${ }^{7}, 12$ ).

Vibratory stimulation partially shows the importance of using motor control systems and providing information for proprioceptive sensibility. Ivanenko et al. reported that walking to an external rhythm increases physical cognitive power of the affected side and improves voluntary control of legs in various situations ${ }^{28}$. Accordingly, in previous studies, gait velocity was improved in patients who received active gait training with vibratory stimulation of the plantar flexors on the affected sides ${ }^{5,15)}$. The mechanism underlying the effects of vibratory stimulation involves an increase in muscle spindle activity ${ }^{29)}$, increase in cortical excitability of the vibrating muscle, and simultaneous reduction of the activity of the antagonists through reciprocal inhibition and upper spinal inhibition ${ }^{30}$ ).

Overall, this study showed that vibratory stimulation of the tibialis anterior muscle during the gait cycle affects general gait parameters such as gait velocity and cadence as well as spatiotemporal gait parameters. Therefore, external cues with vibratory stimulation can prevent foot drop in the swing phase of the gait cycle by contributing to improved control of ankle plantar flexors and can improve gait ability by ensuring the safety of the limbs during the initial contact phase of the gait cycle. Therefore, this technique can be utilized as part of an intensive gait training program to qualitatively improve gait by enhancing voluntary control of the plantar flexors.

Since this cross-sectional study examined only the temporary effects of vibratory stimulation applied to the tibialis anterior muscle on the spatiotemporal gait parameters of chronic stroke patients, further studies are warranted to examine the carryover effects of long-term gait training using the same external cues; in addition, it is necessary to assess whether these effects will be retained for a long period. Furthermore, in the future, further studies must be conducted to analyze treatment effects in terms of biomechanical parameters, and electromyography studies should be performed to examine muscle activity of the tibialis anterior muscle and changes in the start time of muscle contraction in chronic stroke patients under vibratory stimulation conditions.

Taken together, our study showed that the application of vibratory stimulation to the tibialis anterior muscle of chronic stroke patients significantly increased general gait parameters such as gait velocity and cadence and significantly decreased step time, stride time, and the lower limb support rate. The spatial gait parameter, stride length, was 
significantly improved. Therefore, application of external cues with vibratory stimulation of the tibialis anterior muscle during the gait cycle can be utilized as an effective method for gait rehabilitation in chronic stroke patients; that is, it can be used to promote functional activity by controlling gait parameters and improving gait ability.

\section{REFERENCES}

1) Hashidate H, Shiomi T, Sasamoto N: Effects of 6 months combined func tional training on muscle strength, postural balance and gait performance in community-dwelling individuals with chronic stroke hemiplegia. J Phys Ther Sci, 2011, 23: 617-623. [CrossRef]

2) Lord SE, McPherson $\mathrm{K}, \mathrm{McNaughton} \mathrm{HK}$, et al.: Community ambulation after stroke: how important and obtainable is it and what measures appear predictive? Arch Phys Med Rehabil, 2004, 85: 234-239. [Medline] [CrossRef]

3) Stein RB, Everaert DG, Thompson AK, et al.: Long-term therapeutic and orthotic effects of a foot drop stimulator on walking performance in progressive and nonprogressive neurological disorders. Neurorehabil Neural Repair, 2010, 24: 152-167. [Medline] [CrossRef]

4) Ferreira LA, Neto HP, Grecco LA, et al.: Effect of ankle-foot orthosis on gait velocity and cadence of stroke patients: a systematic review. J Phys Ther Sci, 2013, 25: 1503-1508. [Medline] [CrossRef]

5) Kawahira $K$, Higashihara $K$, Matsumoto $S$, et al.: New functional vibratory stimulation device for extremities in patients with stroke. Int J Rehabil Res, 2004, 27: 335-337. [Medline] [CrossRef]

6) Rochester L, Nieuwboer A, Baker K, et al.: The attentional cost of externa rhythmical cues and their impact on gait in Parkinson's disease: effect of cue modality and task complexity. J Neural Transm, 2007, 114: 1243-1248. [Medline] [CrossRef]

7) Amatachaya S, Keawsutthi M, Amatachaya P, et al.: Effects of external cues on gait performance in independent ambulatory incomplete spinal cord injury patients. Spinal Cord, 2009, 47: 668-673. [Medline] [CrossRef]

8) Pelton TA, Johannsen L, Chen H, et al.: Hemiparetic stepping to the beat asymmetric response to metronome phase shift during treadmill gait. Neurorehabil Neural Repair, 2010, 24: 428-434. [Medline] [CrossRef]

9) van Wegen E, de Goede C, Lim I, et al.: The effect of rhythmic somatosensory cueing on gait in patients with Parkinson's disease. J Neurol Sci, 2006, 248: 210-214. [Medline] [CrossRef]

10) Park S, Horak FB, Kuo AD: Postural feedback responses scale with biomechanical constraints in human standing. Exp Brain Res, 2004, 154: 417-427. [Medline] [CrossRef]

11) Sorensen KL, Hollands MA, Patla E: The effects of human ankle muscle vibration on posture and balance during adaptive locomotion. Exp Brain Res, 2002, 143: 24-34. [Medline] [CrossRef]

12) Han J, Kim E, Jung J, et al.: Effect of muscle vibration on spatiotemporal gait parameters in patients with Parkinson's disease. J Phys Ther Sci, 2014, 26: 671-673. [Medline] [CrossRef]

13) McDonough AL, Batavia M, Chen FC, et al.: The validity and reliability of the GAITRite system's measurements: a preliminary evaluation. Arch
Phys Med Rehabil, 2001, 82: 419-425. [Medline] [CrossRef]

14) Patterson KK, Parafianowicz I, Danells CJ, et al.: Gait asymmetry in community-ambulating stroke survivors. Arch Phys Med Rehabil, 2008, 89: 304-310. [Medline] [CrossRef]

15) Paoloni M, Mangone M, Scettri P, et al.: Segmental muscle vibration improves walking in chronic stroke patients with foot drop: a randomized controlled trial. Neurorehabil Neural Repair, 2010, 24: 254-262. [Medline] [CrossRef]

16) Hesse S: Treadmill training with partial body weight support after stroke: a review. NeuroRehabilitation, 2008, 23: 55-65. [Medline]

17) Nadeau $S$, Gravel D, Arsenault AB, et al.: Plantarflexor weakness as a limiting factor of gait speed in stroke subjects and the compensating role of hip flexors. Clin Biomech (Bristol, Avon), 1999, 14: 125-135. [Medline] [CrossRef]

18) Lin PY, Yang YR, Cheng SJ, et al.: The relation between ankle impairments and gait velocity and symmetry in people with stroke. Arch Phys Med Rehabil, 2006, 87: 562-568. [Medline] [CrossRef]

19) Laufer $Y$, Dickstein R, Chefez Y, et al.: The effect of treadmill training on the ambulation of stroke survivors in the early stages of rehabilitation: a randomized study. J Rehabil Res Dev, 2001, 38: 69-78. [Medline]

20) Maki BE: Gait changes in older adults: predictors of falls or indicators of fear. J Am Geriatr Soc, 1997, 45: 313-320. [Medline]

21) Hsu AL, Tang PF, Jan MH: Analysis of impairments influencing gait velocity and asymmetry of hemiplegic patients after mild to moderate stroke. Arch Phys Med Rehabil, 2003, 84: 1185-1193. [Medline] [CrossRef]

22) Titianova EB, Pitkänen K, Pääkkönen A, et al.: Gait characteristics and functional ambulation profile in patients with chronic unilateral stroke. Am J Phys Med Rehabil, 2003, 82: 778-786, quiz 787-789, 823. [Medline] [CrossRef]

23) Balasubramanian CK, Bowden MG, Neptune RR, et al.: Relationship between step length asymmetry and walking performance in subjects with chronic hemiparesis. Arch Phys Med Rehabil, 2007, 88: 43-49. [Medline] [CrossRef]

24) Kottink AI, Hermens HJ, Nene AV, et al.: Therapeutic effect of an implantable peroneal nerve stimulator in subjects with chronic stroke and footdrop: a randomized controlled trial. Phys Ther, 2008, 88: 437-448. [Medline] [CrossRef]

25) Carel C, Loubinoux I, Boulanouar K, et al.: Neural substrate for the effects of passive training on sensorimotor cortical representation: a study with functional magnetic resonance imaging in healthy subjects. J Cereb Blood Flow Metab, 2000, 20: 478-484. [Medline] [CrossRef]

26) Mima T, Sadato N, Yazawa S, et al.: Brain structures related to active and passive finger movements in man. Brain, 1999, 122: 1989-1997. [Medline] [CrossRef]

27) Lotze M, Braun C, Birbaumer N, et al.: Motor learning elicited by voluntary drive. Brain, 2003, 126: 866-872. [Medline] [CrossRef]

28) Ivanenko YP, Grasso R, Lacquaniti F: Influence of leg muscle vibration on human walking. J Neurophysiol, 2000, 84: 1737-1747. [Medline]

29) Rosenkranz K, Rothwell JC: Differential effect of muscle vibration on intracortical inhibitory circuits in humans. J Physiol, 2003, 551: 649-660. [Medline] [CrossRef]

30) Binder C, Kaya AE, Liepert J: Vibration prolongs the cortical silent period in an antagonistic muscle. Muscle Nerve, 2009, 39: 776-780. [Medline] [CrossRef] 